\title{
Boosting Learning Efficacy with Noninvasive Brain Stimulation in Intact and Brain-Damaged Humans
}

\author{
(DFlorian Herpich, ${ }^{1,2,3}$ Michael D. Melnick, ${ }^{4}$ Sara Agosta, ${ }^{1}$ Krystel R. Huxlin, ${ }^{4 \star}$ Doje Tadin, ${ }^{4 *}$ \\ and ${ }^{\circ}$ Lorella Battelli, $1,5,6 *$ \\ ${ }^{1}$ Center for Neuroscience and Cognitive Systems@UniTn, Istituto Italiano di Tecnologia, 38068 Rovereto (TN), Italy, ${ }^{2}$ Center for Mind/Brain Sciences, \\ University of Trento, 38068 Rovereto, Italy, ${ }^{3}$ kbo-Klinikum-Inn-Salzach, 83512 Wasserburg am Inn, Germany, ${ }^{4}$ Department of Brain and Cognitive \\ Sciences, Flaum Eye Institute and Center for Visual Science, University of Rochester, Rochester, New York 14642, ${ }^{5}$ Berenson-Allen Center for Noninvasive \\ Brain Stimulation and Department of Neurology, Beth Israel Deaconess Medical Center, Harvard Medical School, Boston, Massachusetts 02215, and \\ ${ }^{6}$ Cognitive Neuropsychology Laboratory, Harvard University, Cambridge, Massachusetts 02138
}

Numerous behavioral studies have shown that visual function can improve with training, although perceptual refinements generally require weeks to months of training to attain. This, along with questions about long-term retention of learning, limits practical and clinical applications of many such paradigms. Here, we show for the first time in female and male human participants that just $10 \mathrm{~d}$ of visual training coupled with transcranial random noise stimulation (tRNS) over visual areas causes dramatic improvements in visual motion perception. Relative to control conditions and anodal stimulation, tRNS-enhanced learning was at least twice as fast, and, crucially, it persisted for 6 months after the end of training and stimulation. Notably, tRNS also boosted learning in patients with chronic cortical blindness, leading to recovery of motion processing in the blind field after just $10 \mathrm{~d}$ of training, a period too short to elicit enhancements with training alone. In sum, our results reveal a remarkable enhancement of the capacity for long-lasting plastic and restorative changes when a neuromodulatory intervention is coupled with visual training.

Key words: cortical plasticity; noninvasive brain stimulation; perceptual learning; stroke recovery; tRNS; visual areas

\section{Significance Statement}

Our work demonstrates that visual training coupled with brain stimulation can dramatically reduce the training period from months to weeks, and lead to fast improvement in neurotypical subjects and chronic cortically blind patients, indicating the potential of our procedure to help restore damaged visual abilities for currently untreatable visual dysfunctions. Together, these results indicate the critical role of early visual areas in perceptual learning and reveal its capacity for long-lasting plastic changes promoted by neuromodulatory intervention.

\section{Introduction}

The human brain changes throughout life (Li et al., 2004; Gilbert and $\mathrm{Li}, 2012$ ). Visual training is a well known tool for inducing

\footnotetext{
Received Dec. 28, 2018; revised April 10, 2019; accepted May 8, 2019.

Author contributions: F.H., M.D.M., K.R.H., D.T., and L.B. designed research; F.H., M.D.M., and S.A. performed research; F.H., M.D.M., and D.T. analyzed data; K.R.H., D.T., and L.B. wrote the paper.

The present study was funded by the Autonomous Province of Trento, called the "Grandi Progetti 2012," project "Characterizing and improving brain mechanisms of attention-ATTEND (F.H., S.A., L.B.); "Fondazione CaritroBando Ricerca e Sviluppo Economico" (F.H.) and Caritro Bando 2016 "per progetti di ricerca scientifica svolti da giovani ricercatori post-doc" (S.A.); National Institutes of Health (Grants R01-EY-027314 and R01-EY-021209; and CVS training Grant T32-EY-007125), and by an unrestricted grant from the Research to Prevent Blindness Foundation to the Flaum Eye Institute. We thank Valeria Piombino for data collection with neurological patients.

*K.R.H., D.T., and L.B. are coequal senior authors.

K.R.H. is coinventor on U.S. Patent No. 7,549,743 and has founder's equity in Envision Solutions LLC, which licensed this patent from the University of Rochester. The University of Rochester also possesses equity in Envision Solutions LLC. The remaining authors declare no competing financial interests.

Correspondence should be addressed to Lorella Battelli at Lorella.Battelli@iit.it.
}

such changes, improving sensory performance in healthy adults (Sagi, 2011; Li, 2016; Wang et al., 2016; Dosher and Lu, 2017) and in various clinical populations (Deveau et al., 2013; Melnick et al., 2016; Nyquist et al., 2016), a phenomenon referred to as visual perceptual learning (VPL). The specific role of different cortical visual areas during VPL is still openly debated, with several mechanisms likely contributing to learning. For instance, neurophysiological studies have shown that perceptual learning selectively modifies the signal strength of neurons responding to relevant stimulus features, while concurrently suppressing the activity of task-irrelevant information (Yan et al., 2014). Other studies suggest that learning stems from better readout mechanisms in higher-level visual areas (Law and Gold, 2009). Psychophysical studies have suggested that boosting subthreshold, stimulus- 


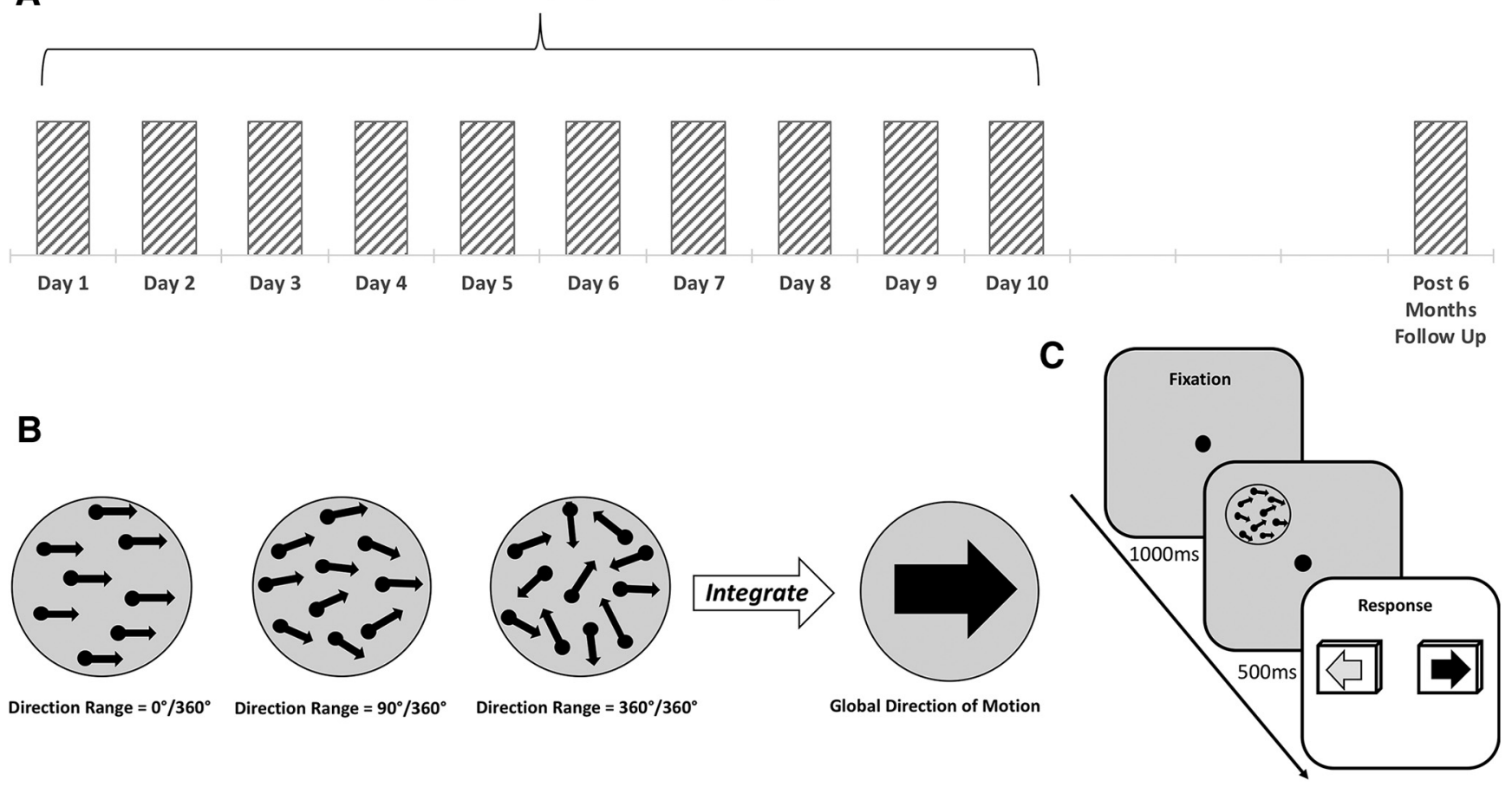

Figure 1. Experimental procedure and behavioral task. A, All participants were tested on a motion integration task to determine baseline performance in the first session (day 1). They then underwent $9 \mathrm{~d}$ of training with or without on-line brain stimulation (days 2-10). Behavioral testing was performed again 6 months after the end of training/stimulation (post-6 month follow-up). $B$, Example of stimuli with different direction ranges $\left(0^{\circ}, 90^{\circ}\right.$, and $\left.360^{\circ}\right)$ used for the motion integration task; target dots were embedded in noise dots that are not shown in the figure for clarity purposes (for details, see Materials and Methods). NDR $=0$ (for details, see text) indicates fully random motion directions ( $360^{\circ}$ range), while NDR $=100$ indicates all signal dots moving in one direction ( $\left(0^{\circ}\right.$ range). A two-alternative forced-choice, adaptive staircase procedure was used to estimate the largest range of dot directions that subjects could correctly integrate to discriminate the global motion direction (leftward vs rightward). C, Trial sequence used for training and to measure left-right motion discrimination thresholds. First, subjects were asked to fixate the central cross for $1000 \mathrm{~ms}$, immediately followed by a tone signaling the appearance of the stimulus, which was presented for $500 \mathrm{~ms}$. Subjects had to indicate the perceived global motion direction by pressing the left or right arrow key on the keyboard.

related cortical activity can promote perceptual learning (Seitz and Dinse, 2007), with attention and reinforcement (provided by reward) increasing stimulus-related neuronal activity and facilitating learning (Ahissar, 2001; Seitz and Watanabe, 2005; Pascucci et al., 2015).

In parallel, increasing effort is being directed at applying visual perceptual training approaches to rehabilitate patients with various types of vision loss, including cortical blindness (CB), amblyopia (Polat et al., 2004; Huang et al., 2008; Levi and Li, 2009; Li et al., 2011, 2013), macular degeneration (Liu et al., 2007; Baker et al., 2008; Kwon et al., 2012), myopia (Tan and Fong, 2008; Camilleri et al., 2014a,b), and even keratoconus (Sabesan et al., 2017). Two critical factors that limit practical applications of VPL are as follows: (1) the long duration of training usually required for adequate performance enhancement (e.g., in patients with chronic CB; Huxlin et al., 2009); and (2) persistence of visual learning and/or recovered abilities after training ends. Noninvasive brain stimulation coupled with perceptual training has emerged as a potentially promising solution for both of these limitations in healthy adults (Fertonani et al., 2011; Falcone et al., 2012; Cappelletti et al., 2013; Sehm et al., 2013; Snowball et al., 2013; Chesters et al., 2017; Zoefel and Davis, 2017).

In $\mathrm{CB}$, a form of vision loss caused by primary visual cortex (V1) damage, one approach shown to recover vision involves training on motion integration tasks in the blind field (Huxlin et al., 2009; Das et al., 2014; Vaina et al., 2014; Cavanaugh and Huxlin, 2017; Fig. 1). However, the training required to restore normal performance on this task in the blind field of patients with
CB typically involves months of daily practice, and is thus difficult to attain and sustain. As such, this represents an ideal task with which to ask whether noninvasive brain stimulation of early visual cortex during training can enhance and speed up the resultant perceptual learning.

We used the following two forms of direct current stimulation to modulate cortical functioning and boost performance during learning: transcranial random noise (tRNS) and anodal transcranial direct current stimulation (a-tDCS).

tRNS was first shown to enhance cortical excitability in the motor cortex (Terney et al., 2008), and subsequent studies reported that it can improve perceptual functions when delivered over the visual cortex (Pirulli et al., 2013; Campana et al., 2014; Tyler et al., 2018; van der Groen et al., 2018), while the effect of a-tDCS is less clear (Miniussi and Ruzzoli, 2013; Ding et al., 2016).

The present experiments asked whether brain stimulation could improve visual learning when administered during training in visually intact humans and whether these improvements persist. We then examined the translational potential of this approach to promote visual recovery in patients with chronic CB. Early visual areas of the brain were targeted for stimulation because of their apparent role in mediating training-induced visual plasticity in physiological, imaging, and brain stimulation studies (Schwartz et al., 2002; Yang and Maunsell, 2004; Rokem and Silver, 2010; Kang et al., 2014; Camilleri et al., 2016; Gratton et al., 2017; Barbot et al., 2018). 


\section{Materials and Methods}

\section{Experiment 1: tRNS-mediated learning in healthy participants}

Regulatory approval. The study was approved by the ethical committee of the University of Trento.

Subjects. A total of 45 subjects participated in the experiment (mean age, 19.9 years; age range, 19-36 years; 32 females and 13 males). All subjects were right handed, neurologically normal, with normal or corrected-to-normal vision and gave written, informed consent before the beginning of the study, according to the ethical standards of the Declaration of Helsinki.

Study design. Subjects were randomly assigned to one of five groups. This included two experimental groups. In the first group, tRNS was delivered over early visual areas (electrodes positioned bilaterally, centered over $\mathrm{O}_{1}$ and $\mathrm{O}_{2}$ of the EEG system coordinates, for the left and right hemisphere respectively). In the second group, a-tDCS was delivered over the occipital cortex (the anode and the cathode were positioned over $\mathrm{Oz}$ and $\mathrm{Cz}$, respectively). While we used bilateral occipital montage for the tRNS condition to match the positioning of other successful studies that found improved performance with tRNS and likely increased excitability in the visual cortex (Romanska et al., 2015; Herpich et al., 2018), particularly with motion discrimination tasks (van der Groen et al., 2018), we chose unilateral montage for the a-tDCS condition, the optimal montage to increase cortical excitability with anodal stimulation of the visual cortex (Antal et al., 2004). Stimulation was concurrent with the training task. There were also three control groups: a sham control; a no-stimulation control; and an active control, where bilateral tRNS was applied over parietal cortex (over P3 and P4, regions likely involved in but not critical for global motion discrimination (Greenlee and Smith, 1997; Battelli et al., 2001). Over $10 \mathrm{~d}$, all subjects were trained to discriminate the left or right global direction of random dot motion stimuli (350 trials/session/d). Day 1 was considered the pretraining session, while day 10 was used as the post-training session (Fig. 1). Finally, a long-term follow-up was performed 6 months after the post-training session. During this follow-up, participants repeated the behavioral baseline tests. Critically, no stimulation was delivered at this time.

Apparatus and procedures. For subjects who underwent brain stimulation, all experiments took place in the same room, under the same light and noise conditions, and with the same apparatus. During each session, participants were positioned on a chinrest/forehead bar combination to stabilize their heads, and to place their eyes $57 \mathrm{~cm}$ from the stimuluspresenting computer monitor. Visual stimuli were generated on a MacBook Pro computer running software based on the Psychophysics Toolbox (Brainard, 1997; Pelli, 1997) in Matlab (MathWorks). Stimuli were presented on a linearized SensEye 3 LED 24 inch monitor (BenQ) with a refresh rate of $120 \mathrm{~Hz}$, and it was luminance calibrated with gamma $=1$ using a professional monitor calibrator (Spyder 5, Datacolor). Eye fixation for all subjects was controlled in real time using an EyeLink 1000 Plus Eye Tracking System (SR Research) whose infrared camera monitored the pupil center and corneal reflection of the left eye. Limits were set so that if the participant's eye moved $>1.5^{\circ}$ in any direction away from the fixation spot during stimulus presentation, loud tones sounded, and the currently displayed trial was aborted and excluded from the final analysis.

Global direction discrimination testing and training. We first measured direction range (DR) thresholds for left-right motion discrimination of circular stimuli that contained a limited percentage of signal dots (Newsome and Paré, 1988; Huxlin and Pasternak, 2004; Levi et al., 2015) and were centered at $\left[-5^{\circ}, 5^{\circ}\right]$ in the visual periphery. To match initial task difficulty across observers, motion coherence (Newsome and Paré, 1988) was calibrated for each subject individually, as previously reported (Levi et al., 2015). The motion coherence of the stimulus was chosen based on preliminary testing aimed to identify a motion signal level that allowed participants to perform the discrimination task just above chance (50\% correct). For all but three subjects, random dot stimuli contained $40 \%$ motion signal. Three subjects were trained with a stimulus containing $30 \%$ coherent motion. Adding coherence noise to our stimuli ensured that all subjects started at about the same difficulty level and, more im- portantly, allowed plenty of room for improvement for our healthy participants. Once a motion signal level was selected for each participant, the task used a QUEST adaptive staircase (Watson and Pelli, 1983) to estimate the broadest distribution of dot directions that subjects could correctly integrate to discriminate the global direction of motion as leftward or rightward. During training, task difficulty was adaptively modulated by adjusting DR of signal dots (Huxlin and Pasternak, 2004) using 12 randomly interleaved 25-trial Quest staircases in each daily session. These adaptive Quest staircases were used to ensure that the stimuli presented to participants are around their threshold performance. Because we collected 300 trials per session, we were able to fit full psychometric functions to the data. Here, we fit single-trial data with the Weibull function (where percentage correct $=1-(1-$ chance $) *$ $\exp \left(-(k * x / \text { threshold })^{\text {slope }}\right)$, and $k=(-\log ((1-0.82) /(1-$ chance $\left.))^{(1 / \text { slope })}\right)$. As customary, we used the $82 \%$ threshold criterion as that is close to the steepest point of the Weibull function. To illustrate the quality of data fits, Figure 2 shows data for an example subject. Thresholds reported in the study, corresponding to $82 \%$ correct, were taken from these estimated Weibull functions and are reported as normalized DR (NDR) thresholds, such that a NDR of $0 \%$ equals fully random motion ( $360^{\circ}$ range of dot directions) and a NDR of $100 \%$ indicates all signal dots moving in one direction $\left(0^{\circ}\right.$ range $)$. The random dot stimuli were presented within a circular aperture $5^{\circ}$ in diameter at a density of 2.6 dots $/{ }^{\circ 2}$. Each dot had a diameter of $0.06^{\circ}$ and moved at a speed of $10 \%$ with a lifetime of $250 \mathrm{~ms}$. The stimulus duration was $500 \mathrm{~ms}$. Each participant started training with DR in the random dot stimulus set to $0^{\circ}$.

The trial sequence was as follows: participants were asked to fixate on a central cross for $1000 \mathrm{~ms}$, immediately followed by a tone signaling the appearance of the stimulus, which was presented for $500 \mathrm{~ms}$. Once the stimulus disappeared, participants had to indicate the perceived global direction of motion by pressing the left or right arrow keys on the keyboard. The two motion directions (leftward and rightward) were randomized across trials. Auditory feedback was provided indicating the correctness of the response on each trial.

During training, stimuli were presented monocularly to the left eye for $10 \mathrm{~d}$ (one session/d from Monday to Friday, for 2 consecutive weeks; Fig. 1 ), while subjects received active, sham, or no stimulation. We chose monocular presentation to closely match the procedure used by Huxlin et al. (2009) on patients and by Levi et al. (2015) on healthy participants using the same visual stimuli. Subjects performed 350 trials/d for a total of 3500 trials by the end of the 2 weeks of training. The total duration of the daily training session for each group was set to last $\sim 20 \mathrm{~min}$.

Stimulation protocols. tDCS and tRNS were delivered using a batterydriven stimulator (DC-Stimulator-Plus, NeuroConn) through a pair of saline-soaked conductive rubber electrodes $\left(35 \mathrm{~cm}^{2}\right)$. Each subject was randomly assigned to one of the five stimulation groups, as described earlier (see Study design). The electrodes were bilaterally placed over the target areas identified following the 10-20 EEG reference system. Subjects wore a Lycra swimmers cap to keep the electrode in place, and we ensured that the skin and hair between the electrodes were completely dry, otherwise preventing the current from reaching the brain. The intensity of stimulation was set to $1.0 \mathrm{~mA}$, and was delivered for $20 \mathrm{~min}$ with a fade in/out period of $20 \mathrm{~s}$. For the a-tDCS group, the polarity of the active electrode was anodal. For the tRNS condition, the random noise stimulation was applied with a $0 \mathrm{~mA}$ offset at frequencies of alternating current ranging from 101 to $640 \mathrm{~Hz}$ (high-frequency tRNS). For the sham stimulation group, the stimulation (using the same electrode montage as in the tRNS condition) was shut down after $20 \mathrm{~s}$. At the end of each session, we asked all subjects to fill out a questionnaire about potential discomfort or any unusual sensation they experienced during the stimulation. Only minor side effects were reported by the tDCS group (two subjects reported slight itching under the electrode, one subject reported a slight subjective temperature increase under the electrode), whereas none of the tRNS group participants reported any sensation of being stimulated.

Data analysis. The Shapiro-Wilk test was used to control for the normality of data distribution. Data sphericity was addressed using Mauchly's test, and a Greenhouse-Geisser correction was used in case of the nonsphericity of the data. Levene's test was used to address the assump- 

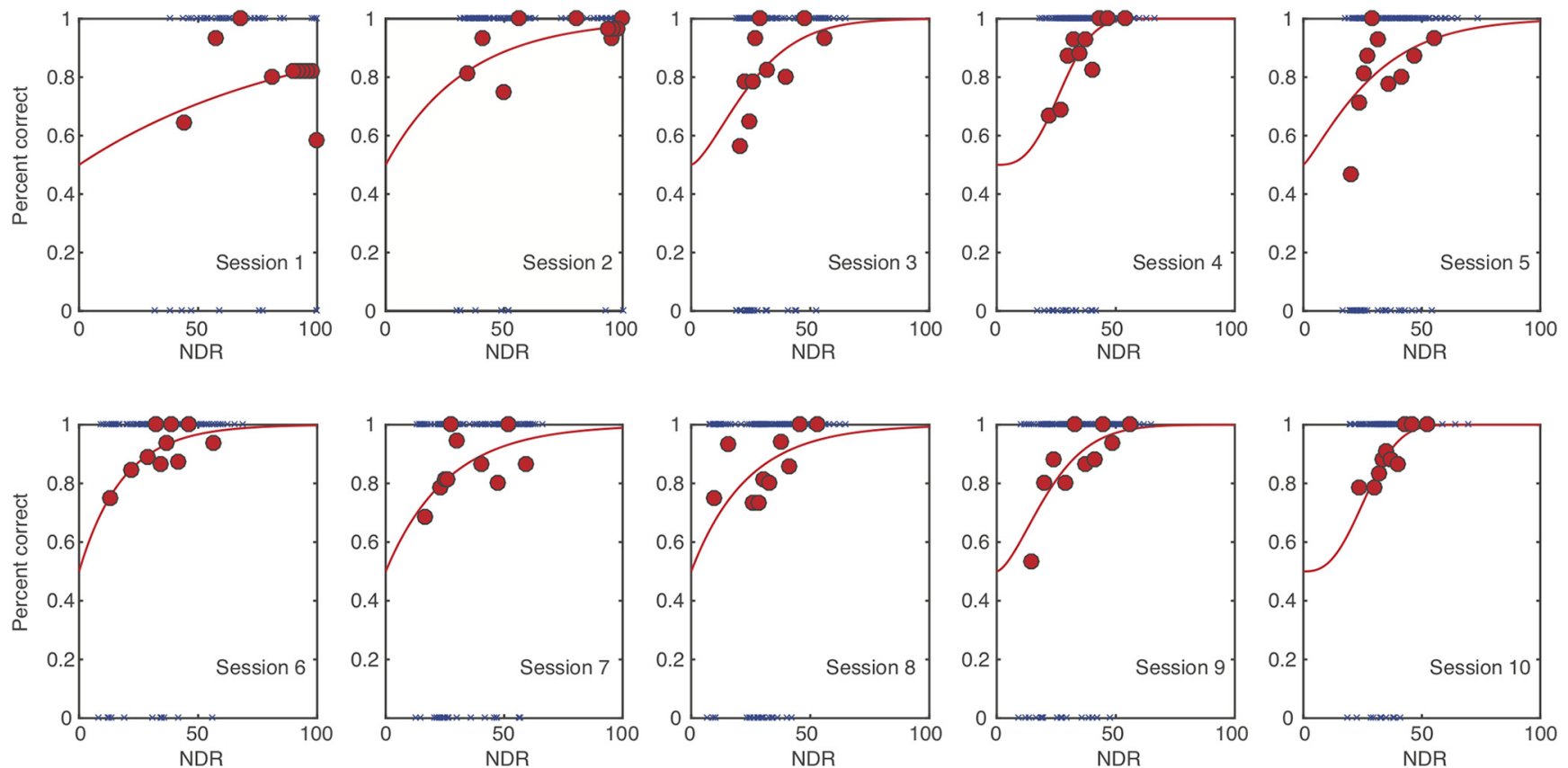

Figure 2. Example psychometric data fits. Here, we selected the tRNS subject whose data were closest to the average of all nine tRNS subjects (going from $94 \%$ NDR to $30 \%$ NDR over 10 sessions). For each session (1-10), blue symbols show all individual trial data (correct trials are at the top and incorrect trials are the bottom of each panel). Psychometric function fits are shown by the red lines. For illustration purposes, individual trials are binned into 1030 -trial bins (red circles).

Table 1. Demographic data for patients with CB

\begin{tabular}{lllll}
\hline Patient & Sex & $\begin{array}{l}\text { Age at testing } \\
\text { (years) }\end{array}$ & Visual defect & Lesion \\
\hline RNS1 & F & 59 & Left lateral homonymous hemianopia & $\begin{array}{l}\text { Time since } \\
\text { lesion (months) }\end{array}$ \\
RNS2 & M & 66 & Left lateral homonymous quadrantanopia & Stroke affecting right posterior capsule-thalamic and occipital lobe \\
RNS3 & M & 53 & Bilateral upper homonymous quadrantanopia & Traumatic brain injury affecting Left fronto-temporo-parietal region \\
Sham1 & M & 69 & Right lateral homonymous hemianopia & Stroke affecting left occipital lobe and left posterior capsule-thalamic area \\
Sham2 & M & 72 & Right lateral homonymous quadrantanopia & Stroke affecting left occipital lobe and internal capsule \\
U1 & F & 63 & Right homonymous hemianopia & Stroke affecting left occipital lobe \\
U2 & F & 67 & Left lower homonymous quadrantanopia & Stroke (hemorrhagic) affecting right occipital lobe \\
U3 & F & 54 & Left homonymous hemianopia & Stroke affecting right occipital cortex \\
U4 & M & 47 & Bilateral hemianopia & Bilateral stroke damage affecting occipital lobes \\
U5 & M & 53 & Left homonymous hemianopia & Stroke affecting right occipital lobe \\
U6 & F & 44 & Left homonymous hemianopia & Stroke affecting right occipital lobe
\end{tabular}

Visual fields defects were assessed with automated perimetry. The last column indicates the time between stroke and in-laboratory testing. Patient RNS3 had a traumatic brain injury, while all others experienced strokes. F, Female; M, male.

tion of equality of variances. The $p$ values were considered significant at $<0.05$. To correct for multiple comparisons in post hoc testing, we used Tukey's HSD correction. The effect sizes are reported as the partial $\eta p^{2}$ values.

\section{Experiment 2: tRNS-mediated visual recovery in patients with cortical blindness}

Regulatory approval. The study on patients was approved by the ethical committee for clinical experimentation of the "Azienda Provinciale per i Servizi Sanitari” (APSS) and by the Institutional Review Board of the University of Rochester Medical Center. The work was conducted after obtaining written, informed consent from each patient.

Cortically blind patients. Eleven patients participated in the study: five recruited at the Center for Neurocognitive Rehabilitation (CeRiN) affiliated to the University of Trento and the Rehabilitation Hospital "Villa Rosa" in Pergine, Italy, and six recruited at the Flaum Eye Institute of the University of Rochester Medical Center. Patients were recruited 2.5-108 months after damage to their early visual areas; as such, all but one patient (U6) were in their chronic, poststroke phase (Table 1; median poststroke time, 15 months). Both the location of damage and the homonymous visual defects were confirmed by neurological reports, neuroradiological examinations, and automated visual perimetry (Optopol
PTS 1000 Visual Field, Canon; or Humphrey Field Analyzer HFA II 750, Carl Zeiss Meditec). The patients gave written, informed consent at their respective study site before participating.

Ten of the patients (RNS1-2, Sham1-2, U1-6) suffered from stroke involving the territory of the posterior cerebral artery, as confirmed by radiological examinations and reports (Table 1, Fig. 3). One patient (RNS3) suffered from traumatic brain injury. Although from the neuroradiological report V1 was not directly affected by trauma, there were indications of visual fields defects, and his visual perimetry showed a clear, homonymous, bilateral upper quadrantanopia; hence, we decided to enroll him in the training procedure (note that data for each patient were computed and shown individually). None of the patients had history or evidence of degenerative or psychiatric disorders. All participants were right handed, with normal or corrected-to-normal visual acuity, and none exhibited visual or other forms of neglect, as determined by neurological examination.

Study design. All cortically blind patients underwent $10 \mathrm{~d}$ of training, following the same day 1 to day 10 procedures as neurotypical subjects (Fig. 1). Patients in Italy were randomly assigned to one of the following two experimental groups: three patients received tRNS over early visual areas during training, whereas two patients received sham stimulation during training, with electrodes placed in the same locations as for tRNS. 

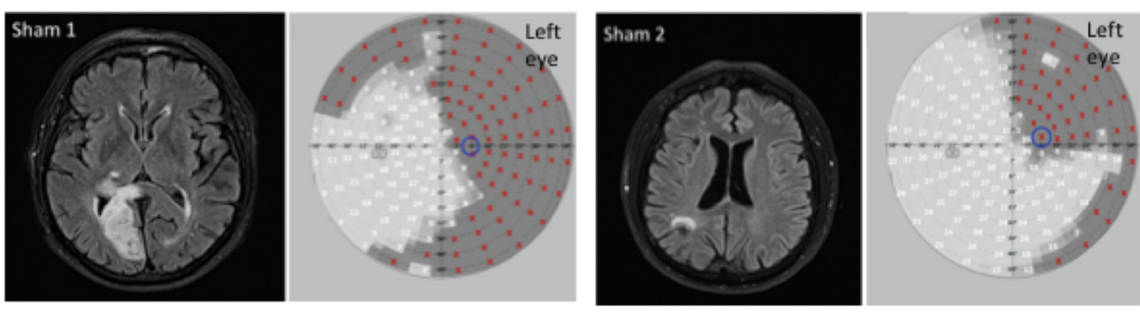

\section{Training Location}
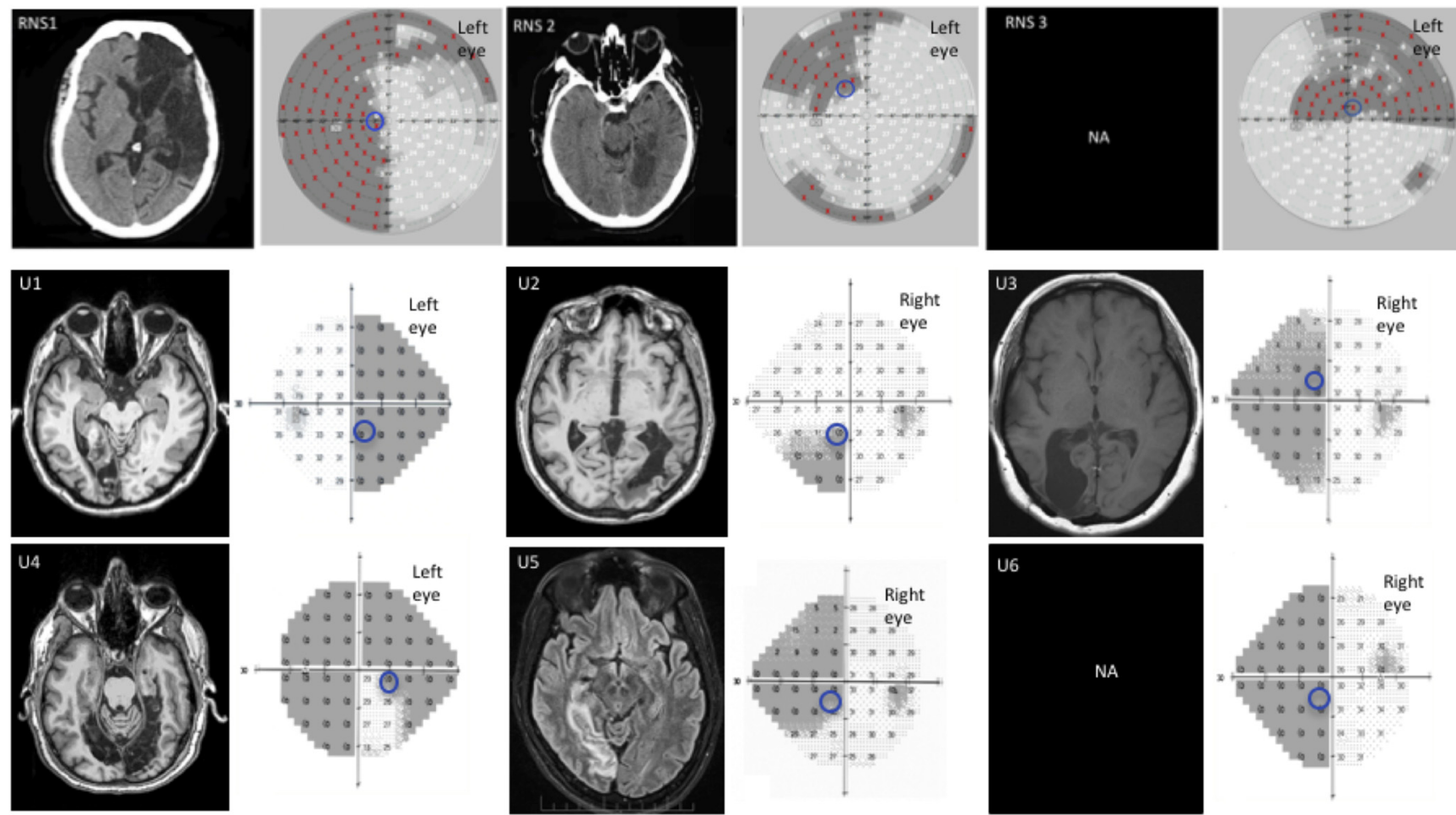

Figure 3. Neuroradiological images and visual perimetries of CB patients. All patients sustained damage of early visual areas or the optic radiations, resulting in homonymous visual field defects as shown by the visual field perimetries, next to each brain image. Within the perimetry images (patients in top two rows: Sham1, Sham2, RNS1, RNS2, and RNS3): red marks and shading areas indicate the patients' blind field. Bottom two rows: Humphrey visual field maps for each of the unstimulated patient (U1-6), with superimposed shading indicating the blind field and numbers indicating the luminance detection sensitivity in the given position expressed in decibels. For all patients, the blue circles indicate the training location and size (for details, see Global direction discrimination testing and training in patients, in Materials and Methods). Radiological images were not available for patients RNS3 and U6.

Patients in the United States underwent global direction discrimination training without brain stimulation.

Apparatus and procedures. We used the same apparatus as in experiment 1 , except that stimuli were presented on a CRT monitor $(48.5 \times$ $31.5 \mathrm{~cm}, 1024 \times 640$ pixel resolution, $120 \mathrm{~Hz}$ frame rate; model $7217 \mathrm{~A}$, HP) calibrated with a ColorCal II automatic calibration system (Cambridge Research Systems) for the patients in the United States. Eye fixation for all patients was controlled in real time using an EyeLink 1000 Plus Eye Tracking System (SR Research).

Global direction discrimination testing and training in patients. First, we spatially mapped motion discrimination performance in each patient to identify a blind field location where training should be performed. We used the same task described in experiment 1 for neurotypical subjects, with the following two adjustments intended to make the task easier for the patients: coherence and NDR were set to $100 \%$ (the easiest possible settings), and the number of trials per training day was lowered to 250 . Fixation was enforced, as in visually intact subjects, and each trial was initiated by fixation of a small circle in the center of the screen. During mapping, stimuli were first presented in the intact field, at locations close to the border with the blind visual field, and patients performed 100 trials of the global direction discrimination task per location. This allowed us to ensure that each patient understood task demands and to assess normal baseline performance on an individual basis. Stimulus location was then moved progressively into the blind field, with 100 trials of the global direction discrimination task performed at each location, until global motion direction discrimination dropped below chance (Huxlin et al., 2009); this was selected as the training location, and care was taken to ensure that it was situated fully inside the perimetrically defined blind field (Fig. 3, blue circles).

For comparison purposes, we also included data from six patients trained in the Huxlin laboratory at the Flaum Eye Institute of the University of Rochester with the same behavioral protocol, but without any brain stimulation. Five of six unstimulated patients, trained with 300 trials/training day, while one trained with 225 trials/d. Thus, on average, unstimulated patients completed $15 \%$ more trials than tRNS/shamstimulated patients, which made them a conservative comparison group.

On Day 1, all patients performed considerably worse on global motion integration in their blind field compared with neurotypical subjects, even at the easiest stimulus level $(100 \%$ coherence; $\mathrm{NDR}=100 \%)$. On day 10 , no patient performed better than $85 \%$ correct at a NDR of $100 \%$ (i.e., with all dots going in the same direction). Specifically, at $100 \%$ coherence, patients' global motion direction range (i.e., NDR) threshold averaged $232 \pm 24.3^{\circ}$ in their intact hemifield and $9.25 \pm 15.91^{\circ}$ in their blind field (paired Student's $t$ test, $p=8.07 \times 10^{5}$ ), indicating that when the global motion was $>9^{\circ}$ around the right or leftward vector none of the subjects were able to discriminate the global direction of motion of the dot stimuli. Thus, given the range of performance by patients, we chose to report the percentage correct at $100 \%$ NDR as the measure of performance. This allowed us to avoid issues with noisy threshold estimates for subthreshold performance, while still retaining a suffi- 
cient dynamic range to capture training-induced improvements in performance.

Stimulation protocols. We performed the exact same stimulation protocols (sham and tRNS) as used with the visually intact participants in experiment 1.

Data analysis. To analyze data from individual patients, we performed the following bootstrap analysis. First, for each subject and each training day, we generated 10,000 bootstrap samples by selecting, with replacement, from the set of available individual trials. Then we fit Weibull functions to all 10,000 samples, and, from the resultant fits, we computed the percentage correct performance at $100 \%$ NDR (the easiest difficulty level). Because most of the individual trials for patients were collected near $100 \%$ NDR, these percentage correct estimates were more robust than threshold estimates, which in many cases were estimated to be $>100 \%$ NDR. Thus, for each training day, we had 10,000 estimates for each patient's percentage correct performance, allowing us to estimate 95\% confidence intervals (see Fig. 5). From this set of estimates, we created 100,000 full datasets for each patient (random sampling with replacement). This allowed us to estimate $p$ values for learning slope and the amount of learning analyses reported in the Results. For the slope analysis, we simply computed the proportion of datasets that had negative learning slopes, multiplying results by 2 to get two-tailed $p$ values. For the amount of learning analysis, we computed the proportion of datasets where day 1-2 performance was better than day 9-10 performance, multiplying results by 2 to get two-tailed $p$ values.

\section{Results}

\section{Impact of pairing brain stimulation with training in visually intact subjects}

Learning of motion integration in control groups

Subjects recruited for the present experiments were divided into five training groups. Two experimental groups (bilateral tRNS and anodal stimulation) received stimulation over early visual areas. Their results were compared with the following three control groups: bilateral tRNS over parietal cortex, a sham control, and a no-stimulation control. Before the onset of training, there were no significant differences in NDR thresholds, a measure of direction integration performance, among the five training groups $\left(F_{(4,40)}=1.15, p=0.35\right)$. This confirmed that all five groups started with relatively similar levels of performance. As expected, all five groups benefited from perceptual training - for each group, performance at day 10 was better than at day 1 (all $t_{(8)}$ $>2.7$, all $p<0.027$ ). This result is consistent with well established effects of training on visual perception (Watanabe and Sasaki, 2015; Levi et al., 2015). However, no significant differences in learning were observed among three of the groups-no stimulation (training only), sham stimulation + training, and tRNS over parietal cortex + training. The lack of difference was observed regardless of whether learning was expressed as a raw change in thresholds $\left(\mathrm{NDR}_{\text {Dayl }}-\mathrm{NDR}_{\text {Dayl } 0} ; F_{(2,24)}=1.58, p=0.23\right)$, a percentage change in thresholds $\left[\left(\mathrm{NDR}_{\text {Dayl }}-\mathrm{NDR}_{\text {Day } 10}\right) / \mathrm{NDR}_{\text {Dayl }}\right.$; $\left.F_{(2,24)}=2.93, p=0.072\right]$ or learning speed (linear regression slope, $\left.F_{(2,24)}=2.36, p=0.12\right)$. We further performed an additional control analysis on the amount of learning between the first and the last session (day 1 to day 10). We ran a repeatedmeasures $t$ test, which showed that the tRNS group (NDR, $56.7 \pm$ 16.2 , mean $\pm S D$ ) differed significantly from each of the following control conditions: sham $\left(34.9 \pm 19.6 ; t_{(8)}=2.57, p=0.01\right)$, behavioral $\left(25.8 \pm 23.6 ; t_{(8)}=-2,19, p=0.02\right)$, and parietal tRNS $\left(21.3 \pm 15.8 ; t_{(8)}=4.68, p<0.001\right)$. To minimize the number of multiple comparisons between experimental and control groups, data from these three control groups were thus combined into a single control dataset for all subsequent analyses.
tRNS, but not a-tDCS, enhances learning

Comparison of the control dataset with tRNS + training and a-tDCS + training revealed large differences in learning (Fig. $4 A, B)$. In addition to the expected main effect of training day $\left(F_{(3.4,144.1)}=34.7, p=10^{-18}\right)$, we found a main effect of group $\left(F_{(2,42)}=3.35, p=0.045\right)$ and, notably, a significant group by day interaction $\left(F_{(6.9,144.1)}=4.01, p=0.01\right)$. As suggested by this significant interaction, the amount of learning differed among the three groups (Fig. $4 A ; F_{(2,42)}=9.39, p=0.0004$ ). Specifically, tRNS + training induced stronger learning than both the combined control group $(p=0.002)$ and a-tDCS + training $(p=$ 0.001; all post hoc tests are Tukey's HSD), whereas a-tDCS outcomes did not differ significantly from those attained by the combined control group ( $p=0.53$ ). The same pattern of results was observed when we considered group differences in terms of the percentage of improvement from pretest to post-test (Fig. $4 B$; $\left.F_{(2,42)}=10.8, p=0.00016\right)$. Again, tRNS + training resulted in larger percentage improvement than that attained by control subjects $(p=0.001)$ and the a-tDCS + training group $(p=$ 0.0002 ). Once again, performance following a-tDCS + training did not differ significantly from that in the combined control group ( $p=0.28$ ). In all groups, learning was well described by a linear trend (Fig. $4 A$ ). Slopes, however, differed among groups (Fig. $4 C ; F_{(2,42)}=7.8, p=0.001$ ), with tRNS + training generating faster learning than in the combined control group ( $p=$ $0.008)$ or a-tDCS + training group $(p=0.001)$. In contrast, a-tDCS did not induce significantly faster learning than that attained by the combined control group $(p=0.30)$.

As tRNS administered during training appeared to cause faster learning, we analyzed at what time point the tRNS group began to diverge from the other two groups. This occurred on day $6\left(F_{(2,42)}=5.03, p=0.01\right)$, at which point the tRNS group showed stronger learning than both the combined control group $(p=0.03$; Tukey's HSD $)$ and the a-tDCS + training group $(p=$ 0.01 ; Tukey's HSD).

In sum, we found strong evidence for enhanced learning in the tRNS + training group, with faster learning than both the combined control group and a-tDCS + training group. As detailed above, this finding was supported regardless of how learning was defined. While it may seem that a-tDCS, as administered in our study, might actually hinder learning (Fig. $4 A, B$ ), this effect was not statistically significant.

\section{Persistence of stimulation-enhanced perceptual learning}

Next, we asked whether the observed enhancement of perceptual learning by tRNS remained stable over an extended period of time. To address this question, we retested participants 6 months after completing the $10 \mathrm{~d}$ of training with and without the different forms of stimulation. The post-test, however, was performed without brain stimulation. The subjects retested at 6 months included 37 of 45 original participants $(n=8$ for tRNS + training group; $n=22$ for the combined control group; and $n=7$ for a-tDCS + training group). Figure $4 D$ contrasts the amount of learning at day $10\left(\mathrm{NDR}_{\text {Day } 1}-\mathrm{NDR}_{\text {Day 10 }}\right)$ with that exhibited 6 months after the end of training $\left(\mathrm{NDR}_{\text {Day } 1}-\mathrm{NDR}_{6 \text {-months }}\right)$. There was a small, nonsignificant loss in performance for the three groups (no main effect of testing day; $F_{(1,34)}=3.32, p=0.8$ ) and no interaction $\left(F_{(2,34)}=0.88, p=0.43\right)$. We only found a main effect of group, confirming that the group differences at the end of $10 \mathrm{~d}$ of training remained unaltered 6 months after training $\left(F_{(2,34)}=4.68, p=0.02\right)$. Thus, it appears that tRNS enhanced perceptual learning over the long term - at least 6 months after the end of both training and brain stimulation. Moreover, 
A

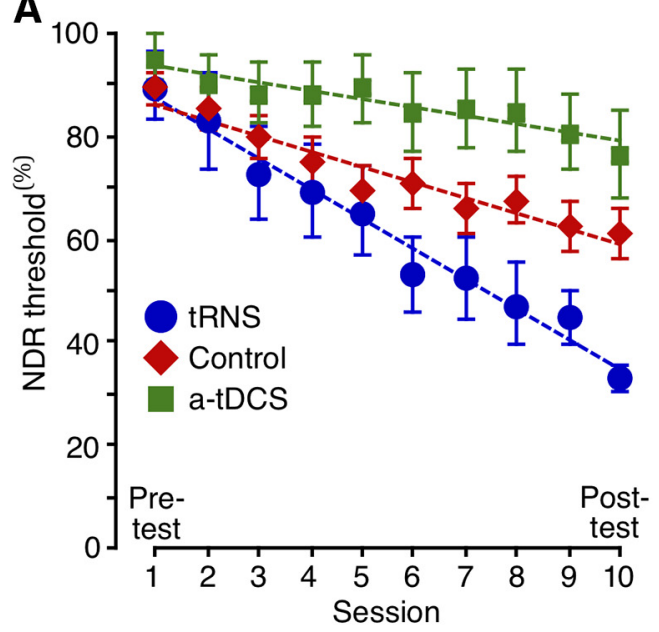

C

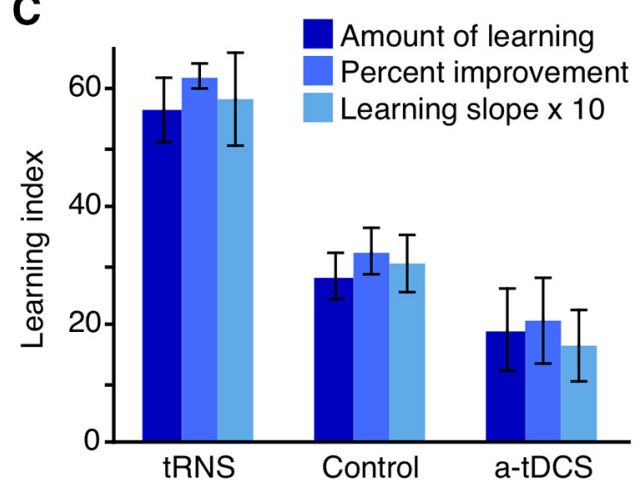

B
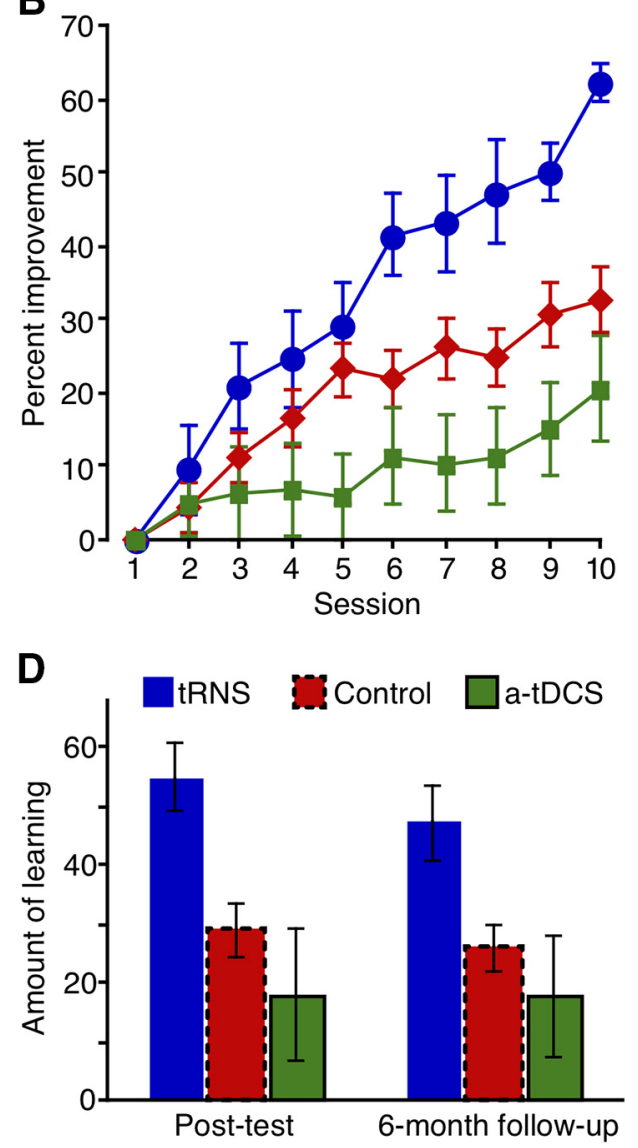

Figure 4. Effects of brain stimulation on perceptual learning in visually intact subjects. $\boldsymbol{A}$, NDR thresholds for the control groups, tRNS group, and a-tDCS group. Dashed lines are linear fits, indicating the learning slope. $\boldsymbol{B}$, Same data as in $\boldsymbol{A}$, but expressed as the percentage improvement relative to day 1 thresholds. $\boldsymbol{C}$, Learning index computed in three different ways. The tRNS group exhibited a significantly stronger amount of learning (day $1-$ day $10 ; F_{(2,42)}=9.39, p=0.0004$; all Tukey's HSD, $\left.p<0.002\right)$, percentage improvement $(100 *$ (day $1-$ day 10$) /$ day $1 ; F_{(2,42)}=$ $10.8, p=0.00016$; all Tukey's HSD, $p<0.001)$, and learning slope $\left(F_{(2,42)}=7.8, p=0.001\right.$; all Tukey's HSD, $\left.p<0.008\right)$ than both the control and a-tDCS groups. $D$, Amount of learning, defined as the difference from day 1 thresholds, at the end of the training (left) and 6 months after (right). Error bars are \pm 1 SEM.

this persistent enhancement was observed without brain stimulation at the 6 month follow-up. This suggested that the enhancement of perceptual learning by tRNS was not due to on-line or short-term optimization of visual processing, but instead, resulted in consolidated sensory learning.

\section{tRNS boosts training-induced visual recovery in cortically blind patients}

Given our finding that tRNS, but not a-tDCS, over occipital cortex considerably enhances perceptual learning in neurotypical subjects, we next asked whether tRNS is also able to enhance training-induced visual recovery in chronic, V1-damaged patients with CB. To the best of our knowledge, tRNS has not been attempted in brain-damaged patients. Moreover, whether tRNS over early visual areas could enhance learning in CB patients is an open question, as learning in this patient population can exhibit properties not found in neurotypical subjects (Das et al., 2014; Vaina et al., 2014; Cavanaugh and Huxlin, 2017), and since, by definition, the part of early visual cortex that would normally be stimulated is damaged. Hence, we sought to perform a preliminary, proof-of-concept study in five patients with occipital damage resulting in homonymous visual field defects measured with visual perimetry (Table 1, Fig. 3).

Visual perimetry was used to identify the blind field borders and select training locations in the blind field (Fig. 3). We ran- domly assigned five patients from our Italian study site to either tRNS + training $(n=3$, RNS1-3) or sham stimulation + training $(n=2$, Sham 1-2). Data from an additional six patients with CB who trained identically, but without brain stimulation (unstimulated, U1-6), at our U.S. study site, were also analyzed for comparison. All patients, at both study sites, trained for $10 \mathrm{~d}$ using random dot stimuli, as in neurotypical subjects (Fig. 1). Because patients have difficulty seeing motion, their stimuli, unlike those for neurotypical subjects, did not include noise dots.

As expected (Huxlin et al., 2009; Das et al., 2014; Cavanaugh et al., 2016; Cavanaugh and Huxlin, 2017), all patients performed considerably worse on global motion integration in their blind field compared with neurotypical subjects. This was the case even at the easiest stimulus level (NDR $=100$, with all dots moving in the same direction), where none of the patients exhibited ceiling level performance in their blind field. As such, we used the percentage correct at $100 \mathrm{NDR}$ as the measure of performance (for details, see Materials and Methods). Sham-stimulated patients exhibited no significant change in performance across the $10 \mathrm{~d}$ of training (Fig. 5A), as evidenced by learning slopes that were not significantly different from 0 (all $p>0.63$; for bootstrap procedure used to analyze data from individual patients, see Materials and Methods). This was comparable to the lack of learning observed in the six unstimulated patients, who also did not exhibit a significant learning slope over their first $10 \mathrm{~d}$ of training (Fig. 5C; 

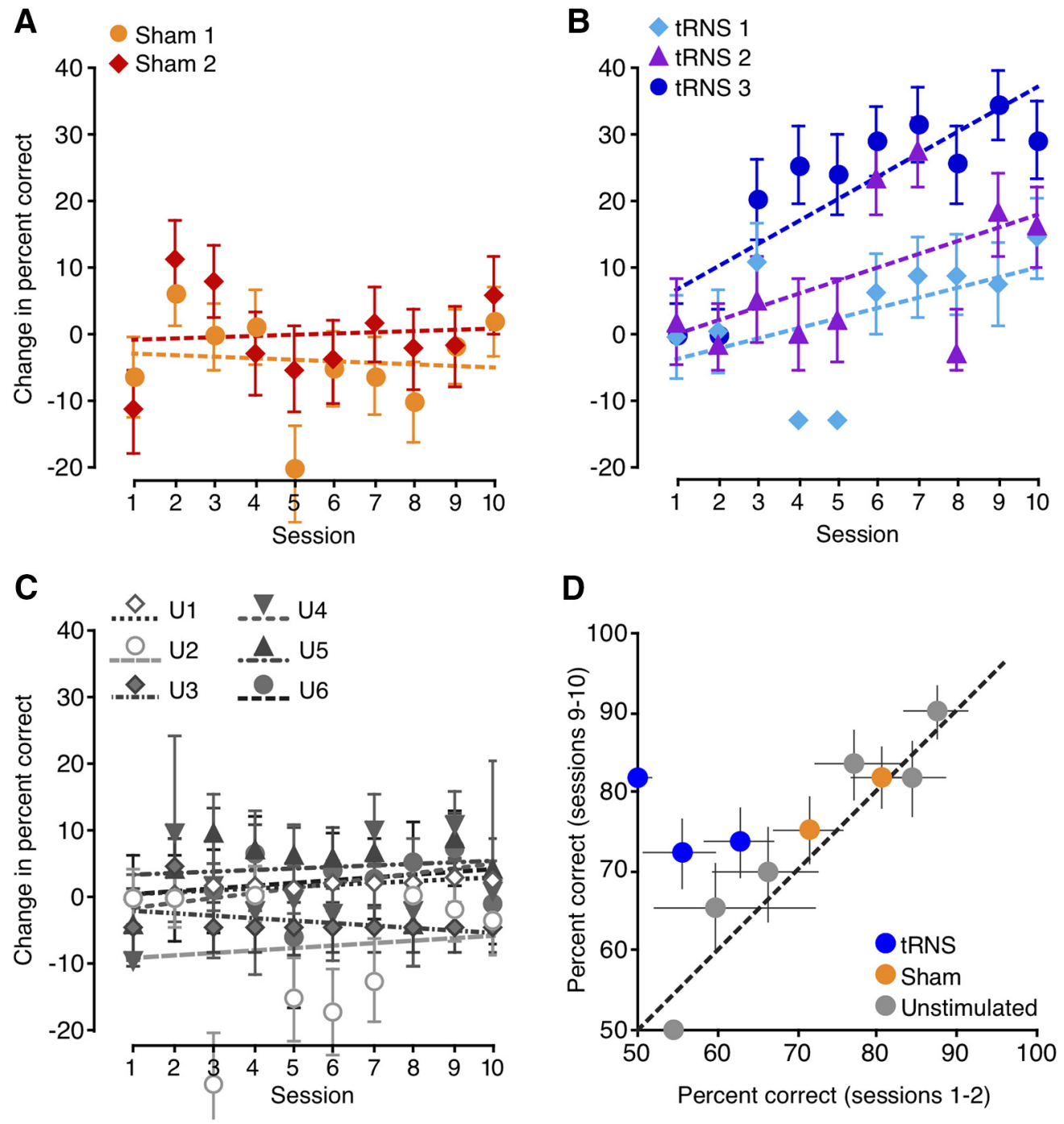

Figure 5. The effects of brain stimulation on perceptual learning in patients with $(B$. $A-C$, Task performance over 10 training days for patients who underwent sham stimulation $(A)$, those who received tRNS $(\boldsymbol{B})$, and six unstimulated patients $(\boldsymbol{C})$. The raw percentage of correct performance was normalized by subtracting the average percentage correct for the first 2 training days. $\boldsymbol{D}$, Comparison of the raw percentage correct averaged over the first $2 \mathrm{~d}$ against the raw percentage correct for the final 2 training days. Significant learning was observed only for patients who trained with tRNS. All error bars indicate $95 \%$ confidence intervals. For $\boldsymbol{A}-\boldsymbol{C}$, all lines are linear fits, indicating the learning slope.

all $p>0.13$ ). In contrast, tRNS coupled with training enhanced the rate of global motion discrimination learning in $\mathrm{CB}$ patients (Fig. $5 B$ ), who exhibited significantly positive learning slopes (all $p<0.0048)$. We also examined the change in performance from days 1-2 to days $9-10$, averaging results over 2 consecutive days to minimize the effects of day-by-day fluctuations. The analysis showed significant change only for patients trained with tRNS (Fig. 5D; tRNS, all $p<0.0002$; sham, all $p>0.24$; unstimulated, all $p>0.080)$.

\section{Discussion}

In the present study, we asked whether brain stimulation over early visual cortex could boost and speed up visual perceptual learning in healthy humans and in those affected by cortically induced blindness. In healthy humans, we also asked whether enhancements attained from training coupled with stimulation persisted over long periods. Results from experiment 1 show that tRNS applied bilaterally over healthy, early visual cortex speeds up and boosts performance during visual perceptual learning. Over $10 \mathrm{~d}$, neurotypical subjects in the tRNS + training group exhibited an $\sim 60 \%$ improvement in motion integration thresh- olds (Fig. 4B), which was two and three times as strong as learning attained by the control and a-tDCS groups, respectively. This finding was supported regardless of whether learning was defined as a raw change in NDR threshold, a percentage improvement, or the slope of a linear fit to the data. The observed benefit of tRNS over training alone or sham stimulation + training, or tDCS over parietal cortex is consistent with evidence that tRNS is especially effective at promoting plasticity when coupled with a relevant stimulus, and when it is applied over relevant (in our case, occipital) brain areas (Cappelletti et al., 2013). In contrast, we found no such benefit of a-tDCS over occipital cortex. Finally, we provide for the first time preliminary evidence that $\mathrm{RNS}$ enhances vision recovery in patients with $\mathrm{CB}$ with $\mathrm{V} 1$ damage. Moreover, we demonstrate seemingly safe usage of this technique in a class of stroke patients, with no side effects reported. Notably, with respect to training-induced recovery, tRNS enabled improvements in visual task performance of patients with chronic CB in their blind field, over a tiny fraction of the training days typically required to induce such improvements in the absence of brain stimulation (Huxlin et al., 2009; Das and Huxlin, 2010; Das et al., 
2014; Melnick et al., 2016; on average, 72-80 training days are required to recover global direction discrimination performance at a given blind field location (Cavanaugh and Huxlin, 2017). Indeed, we observed a steady and significant increase in performance for three patients trained with tRNS over $10 \mathrm{~d}$, with no such effects in patients who trained with sham stimulation. $\mathrm{Pa}$ tients with chronic CB are a population that would especially benefit from enhanced perceptual learning because vision recovery using conventional training methods usually takes many months of daily training (Huxlin et al., 2009; Das and Huxlin, 2010; Das et al., 2014; Melnick et al., 2016; Cavanaugh and Huxlin, 2017).

Interestingly, while the position of the stimulating electrodes corresponded to early visual areas that included V1, improvement occurred despite $\mathrm{V} 1$ being damaged in the patients with $\mathrm{CB}$. This might suggest that the neuromodulatory benefit of tRNS likely impacts any residual V1, but also adjacent visual areas such as V2 and V3, which might have also supported visual learning, as indicated in imaging studies in CB patients (Henriksson et al., 2007; Raemaekers et al., 2011; Martin et al., 2012; Ajina et al., 2015; Barbot et al., 2018). V2 and V3 are usually spared in CB patients, and they might have played a pivotal role in supporting recovery during training. We cannot address whether some form of cortical reorganization took place in 2 weeks of training in our patients, as we did not collect any imaging data. However, we could speculate that since we delivered bilateral stimulation, this either boosted cortical functioning within the affected hemisphere, promoting activity in the damaged early visual cortex in response to concurrent visual/brain stimulation or, alternatively, visual areas in the healthy hemisphere, homologs to the damaged areas, might have promoted recovery (Henriksson et al., 2007). These remain interesting hypotheses to be further tested in future studies. Interestingly, physiological studies have shown that V2/V3 inactivation may degrade the ability of cortical motionsensitive areas to work efficiently (Ponce et al., 2011), while they might support learning when they function normally (Law and Gold, 2008). In summary, while plasticity of spared visual circuits is generally believed to underlie visual recovery, the specific neural mechanisms involved remain unclear.

An additional finding in the present study was the observation that the enhancement of perceptual learning induced by tRNS can persist over an extended period of time (6 months in the present study) beyond the end of stimulation and training. This is important since stimulation-enhanced perceptual learning would have limited practical use if its beneficial effects diminished over time. Additionally, because all subjects performed the behavioral task with no stimulation at the follow-up, 6 month time point, we infer that the benefits of tRNS go beyond an online enhancement of visual processing and likely involve plastic changes that persist within the visual system, allowing it to more effectively process global motion stimuli. As such, we conclude that consolidation of learning occurred in our subjects. The persistence effects observed here are particularly notable, as many VPL studies failed to see long-lasting effects and/or the transfer of learning to other tasks (Dosher and Lu, 2017).

Some important questions arising from the present results are as follows: (1) how does brain stimulation enhance the effects of perceptual learning, and (2) why do some forms of stimulation prove effective and others not? a-tDCS did not exert a beneficial effect in our study-a surprising result given the results from previous studies stimulating the early visual cortex (Antal et al., 2004). One possible explanation is that the strongest effects of tDCS were reported off-line, where a-tDCS was delivered prior to the measured behavior (Pirulli et al., 2013). Another possibility is that a-tDCS is not the ideal neuromodulatory technique for repeated sessions. Although it alters membrane potentials and hence exerts increased excitability, it may also engage inhibitory homeostatic mechanisms during repeated sessions (Fertonani et al., 2011; Peters et al., 2013).

Our observation that visual enhancements persist long after both training and tRNS ended does constrain the possible mechanistic explanations. Multiple types of transcranial electrical stimulation have been shown to alter excitability in cortex, and the longer time course of direct current stimulation effects has been suggested to relate to homeostatic changes in membrane potential (Liebetanz et al., 2002; Nitsche et al., 2003; Ardolino et al., 2005; Terney et al., 2008) or gate threshold (Bikson et al., 2013). However, direct evidence that transcranial stimulation alters the dynamics of networks known to be related to perceptual learning, such as dopaminergic reward networks (Seitz and Watanabe, 2005; Pascucci et al., 2015), has not yet been provided. All we can state here is that tRNS does not appear to globally affect reward networks, as there was no boost in visual performance or learning seen from stimulation over parietal cortex.

An interesting hypothesis is that tRNS-related visual performance improvements might derive from the state of the neurons at the time of stimulation (Silvanto et al., 2008), and that adding noise to the cortex might enhance sensory detection, in particular when stimuli are presented at threshold and embedded in noise (Abrahamyan et al., 2015). Several short-term mechanisms have also been proposed to explain the effects of tRNS, and a favored hypothesis involves stochastic resonance, whereby random frequency stimulation in tRNS appears to boost the responses of neural populations to weak inputs; thus, the stochastic effect is expected to be highest for stimuli presented below or just above threshold, as in our task (Moss et al., 2004; Schwarzkopf et al., 2011; Miniussi and Ruzzoli, 2013; Pirulli et al., 2013; van der Groen and Wenderoth, 2016; Herpich et al., 2018; van der Groen et al., 2018). An alternative hypothesis proposes temporal summation of excitatory signals between visual stimulation and electrical stimulation (Terney et al., 2008; Fertonani et al., 2011; Pirulli et al., 2013), and selective enhancement of active neural networks (Bikson et al., 2013; Miniussi et al., 2013; Luft, 2014; Fertonani and Miniussi, 2017). It is important to note that one or more of these short-term mechanisms may be the first step in a longer-term cascade that results in a persistence of learning. For instance, stronger activation of task-relevant neurons due to temporal summation or stochastic resonance may encourage a shift toward greater plasticity in sensory processing and/or readout. However, the time course of effects observed in the present study, and especially their persistence, suggests that on-line phenomena (i.e., during stimulation or shortly thereafter) are not the only ones at play with respect to learning enhancements induced by tRNS. Interestingly, studies on perceptual learning in animal models have shown that learning might boost the modulation in neuronal tuning to stimulus components relevant to the task (Liu and Pack, 2017). If learning was associated with changes in the tuning characteristics of neurons (for review, see Gilbert and $\mathrm{Li}$, 2012), we could speculate that tRNS coupled with behavioral training might facilitate and consolidate this plastic change, which could then persist across months (Snowball et al., 2013).

Regardless of its precise mechanism of action, here we provide empirical evidence for the potential usefulness of tRNS coupled with visual training on a patient population that requires perceptual learning to attain visual recovery. V1-damaged patients with chronic $\mathrm{CB}$ are able to recover some visual abilities within their 
scotoma, but only after intensive and repetitive training over many months of daily practice (Huxlin et al., 2009; Das et al., 2014). The application of safe, painless neurostimulation in situations like this, where perceptual learning is directly proportional to the quantity of vision recovered (Cavanaugh and Huxlin, 2017), has the potential to dramatically improve quality of life and treatment outcomes (Cavanaugh et al., 2016). Therefore, results from our experiment with $\mathrm{CB}$ patients suggest that tRNS might be a viable adjunct procedure to speed up the recovery process. Remarkably, even though the physiological effects of tRNS upon the damaged early visual cortex are currently unknown, our data show that tRNS can help overcome reduced and/or partially absent functionality and boost learning in the blind field.

\section{References}

Abrahamyan A, Clifford CW, Arabzadeh E, Harris JA (2015) Low intensity TMS enhances perception of visual stimuli. Brain Stimul 8:1175-1182.

Ahissar M (2001) Perceptual training: a tool for both modifying the brain and exploring it. Proc Natl Acad Sci U S A 98:11842-11843.

Ajina S, Pestilli F, Rokem A, Kennard C, Bridge H (2015) Human blindsight is mediated by an intact geniculo-extrastriate pathway. Elife 4:e08935.

Antal A, Kincses TZ, Nitsche MA, Bartfai O, Paulus W (2004) Excitability changes induced in the human primary visual cortex by transcranial direct current stimulation: direct electrophysiological evidence. Invest Ophthalmol Vis Sci 45:702-707.

Ardolino G, Bossi B, Barbieri S, Priori A (2005) Non-synaptic mechanisms underlie the after-effects of cathodal transcutaneous direct current stimulation of the human brain. J Physiol 568:653-663.

Baker CI, Dilks DD, Peli E, Kanwisher N (2008) Reorganization of visual processing in macular degeneration: replication and clues about the role of foveal loss. Vision Res 48:1910-1919.

Barbot A, Melnick M, Cavanaugh M, Das A, Merriam E, Heeger D, Huxlin KR (2018) Visual recovery in chronic cortically-blind patients relies on spared cortical activity and increased V1 coverage of the blind field (abstract). J Vis 18:1074.

Battelli L, Cavanagh P, Intriligator J, Tramo MJ, Hénaff MA, Michèl F, Barton JJ (2001) Unilateral right parietal damage leads to bilateral deficit for high-level motion. Neuron 32:985-995.

Bikson M, Name A, Rahman A (2013) Origins of specificity during tDCS: anatomical, activity-selective, and input-bias mechanisms. Front Hum Neurosci 7:688.

Brainard DH (1997) The psychophysics toolbox. Spat Vis 10:433-436.

Camilleri R, Pavan A, Ghin F, Battaglini L, Campana G (2014a) Improvement of uncorrected visual acuity and contrast sensitivity with perceptual learning and transcranial random noise stimulation in individuals with mild myopia. Front Psychol 5:1234.

Camilleri R, Pavan A, Ghin F, Campana G (2014b) Improving myopia via perceptual learning: is training with lateral masking the only (or the most) efficacious technique? Atten Percept Psychophys 76:2485-2494.

Camilleri R, Pavan A, Campana G (2016) The application of on-line transcranial random noise stimulation and perceptual learning in the improvement of visual functions in mild myopia. Neuropsychologia 89:225-231.

Campana G, Camilleri R, Pavan A, Veronese A, Lo Giudice G, Lo (2014) Improving visual functions in adult amblyopia with combined perceptual training and transcranial random noise stimulation (tRNS): a pilot study. Front Psychol 5:1402.

Cappelletti M, Gessaroli E, Hithersay R, Mitolo M, Didino D, Kanai R, Cohen Kadosh R, Walsh V (2013) Transfer of cognitive training across magnitude dimensions achieved with concurrent brain stimulation of the parietal lobe. J Neurosci 33:14899-14907.

Cavanaugh MR, Huxlin KR (2017) Visual discrimination training improves humphrey perimetry in chronic cortically induced blindness. Neurology 88:1856-1864.

Cavanaugh MR, Lilley S, Melnick MD, Reisner A, Huxlin KR (2016) Visual discrimination training shrinks cortically blind fields and improves quality of life in chronic stroke patients (abstract). J Vis 16:31.

Chesters J, Watkins KE, Möttönen R (2017) Investigating the feasibility of using transcranial direct current stimulation to enhance fluency in people who stutter. Brain Lang 164:68-76.
Das A, Huxlin KR (2010) New approaches to visual rehabilitation for cortical blindness: outcomes and putative mechanisms. Neuroscientist 16:374-387.

Das A, Tadin D, Huxlin KR (2014) Beyond blindsight: properties of visual relearning in cortically blind fields. J Neurosci 34:11652-11664.

Deveau J, Lovcik G, Seitz AR (2013) The therapeutic benefits of perceptual learning. Curr Trends Neurol 7:39-49.

Ding Z, Li J, Spiegel DP, Chen Z, Chan L, Luo G, Yuan J, Deng D, Yu M, Thompson B (2016) The effect of transcranial direct current stimulation on contrast sensitivity and visual evoked potential amplitude in adults with amblyopia. Sci Rep 6:19280.

Dosher B, Lu ZL (2017) Visual perceptual learning and models. Annu Rev Vis Sci 3:343-363.

Falcone B, Coffman BA, Clark VP, Parasuraman R (2012) Transcranial direct current stimulation augments perceptual sensitivity and 24-hour retention in a complex threat detection task. PLoS One 7:e34993.

Fertonani A, Miniussi C (2017) Transcranial electrical stimulation: what we know and do not know about mechanisms. Neuroscientist 23:109-123.

Fertonani A, Pirulli C, Miniussi C (2011) Random noise stimulation improves neuroplasticity in perceptual learning. J Neurosci 31:1541615423.

Gilbert CD, Li W (2012) Adult visual cortical plasticity. Neuron 75:250264.

Gratton C, Yousef S, Aarts E, Wallace DL, D'Esposito M, Silver MA (2017) Cholinergic, but not dopaminergic or noradrenergic, enhancement sharpens visual spatial perception in humans. J Neurosci 37:4405-4415.

Greenlee MW, Smith AT (1997) Detection and discrimination of first- and second-order motion in patients with unilateral brain damage. J Neurosci 17:804-818.

Henriksson L, Raninen A, Näsänen R, Hyvärinen L, Vanni S (2007) Training-induced cortical representation of a hemianopic hemifield. J Neurol Neurosurg Psychiatry 78:74-81.

Herpich F, Contò F, van Koningsbruggen M, Battelli L (2018) Modulating the excitability of the visual cortex using a stimulation priming paradigm. Neuropsychologia 119:165-171.

Huang CB, Zhou Y, Lu ZL (2008) Broad bandwidth of perceptual learning in the visual system of adults with anisometropic amblyopia. Proc Natl Acad Sci U S A 105:4068-4073.

Huxlin KR, Pasternak T (2004) Training-induced recovery of visual motion perception after extrastriate cortical damage in the adult cat. Cereb Cortex 14:81-90.

Huxlin KR, Martin T, Kelly K, Riley M, Friedman DI, Burgin WS, Hayhoe M (2009) Perceptual relearning of complex visual motion after V1 damage in humans. J Neurosci 29:3981-3991.

Kang JI, Huppé-Gourgues F, Vaucher E (2014) Boosting visual cortex function and plasticity with acetylcholine to enhance visual perception. Front Syst Neurosci 8:172.

Kwon M, Ramachandra C, Satgunam P, Mel BW, Peli E, Tjan BS (2012) Contour enhancement benefits older adults with simulated central field loss. Optom Vis Sci 89:1374-1384.

Law CT, Gold JI (2008) Neural correlates of perceptual learning in a sensory-motor, but not a sensory, cortical area. Nat Neurosci 11:505-513.

Law CT, Gold JI (2009) Reinforcement learning can account for associative and perceptual learning on a visual-decision task. Nat Neurosci 12: 655-663.

Levi A, Shaked D, Tadin D, Huxlin KR (2015) Is improved contrast sensitivity a natural consequence of visual training? J Vis 15:4.

Levi DM, Li RW (2009) Perceptual learning as a potential treatment for amblyopia: a mini-review. Vision Res 49:2535-2549.

Li J, Thompson B, Deng D, Chan LY L, Yu M, Hess RF (2013) Dichoptic training enables the adult amblyopic brain to learn. Curr Biol 23:R308 R309.

Li RW, Ngo C, Nguyen J, LeviDM (2011) Video-game play induces plasticity in the visual system of adults with amblyopia. PLoS Biol 9:e1001135.

Li W (2016) Perceptual learning: use-dependent cortical plasticity. Annu Rev Vis Sci 2:109-130.

Li W, Piëch V, Gilbert CD (2004) Perceptual learning and top-down influences in primary visual cortex. Nat Neurosci 7:651-657.

Liebetanz D, Nitsche MA, Tergau F, Paulus W (2002) Pharmacological approach to the mechanisms of transcranial DC-stimulation-induced aftereffects of human motor cortex excitability. Brain 125:2238-2247. 
Liu L, Kuyk T, Fuhr P (2007) Visual search training in subjects with severe to profound low vision. Vision Res 47:2627-2636.

Liu LD, Pack CC (2017) The contribution of area MT to visual motion perception depends on training. Neuron 95:436-446.e3.

Luft CD (2014) Learning from feedback: the neural mechanisms of feedback processing facilitating better performance. Behav Brain Res 261:356-368.

Martin T, Das A, Huxlin KR (2012) Visual cortical activity reflects faster accumulation of information from cortically blind fields. Brain 135: $3440-3452$.

Melnick MD, Tadin D, Huxlin KR (2016) Relearning to see in cortical blindness. Neuroscientist 22:199-212.

Miniussi C, Ruzzoli M (2013) Transcranial stimulation and cognition. In: Handbook of clinical neurology, Vol 116, Ed 1, pp 739-750. Amsterdam: Elsevier.

Miniussi C, Harris JA, Ruzzoli M (2013) Modelling non-invasive brain stimulation in cognitive neuroscience. Neurosci Biobehav Rev 37: $1702-1712$

Moss F, Ward LM, Sannita WG (2004) Stochastic resonance and sensory information processing: a tutorial and review of application. Clin Neurophysiol 115:267-281.

Newsome WT, Paré EB (1988) A selective impairment of motion perception following lesions of the middle temporal visual area (MT). J Neurosci 8:2201-2211.

Nitsche MA, Liebetanz D, Antal A, Lang N, Tergau F, Paulus W (2003) Modulation of cortical excitability by weak direct current stimulationtechnical, safety and functional aspects. Suppl Clin Neurophysiol. 56:255-276.

Nyquist JB, Lappin JS, Zhang R, Tadin D (2016) Perceptual training yields rapid improvements in visually impaired youth. Sci Rep 6:37431.

Pascucci D, Mastropasqua T, Turatto M (2015) Monetary reward modulates task-irrelevant perceptual learning for invisible stimuli. PLoS One 10:e0124009.

Pelli DG (1997) The VideoToolbox software for visual psychophysics: transforming numbers into movies. Spat Vis 10:437-442.

Peters MA, Thompson B, Merabet LB, Wu AD, Shams L (2013) Anodal tDCS to V1 blocks visual perceptual learning consolidation. Neuropsychologia 51:1234-1239.

Pirulli C, Fertonani A, Miniussi C (2013) The role of timing in the induction of neuromodulation in perceptual learning by transcranial electric stimulation. Brain Stimul 6:683-689.

Polat U, Ma-Naim T, Belkin M, Sagi D (2004) Improving vision in adult amblyopia by perceptual learning. Proc Natl Acad Sci U S A 101:66926697.

Ponce CR, Hunter JN, Pack CC, Lomber SG, Born RT (2011) Contributions of indirect pathways to visual response properties in macaque middle temporal area MT. J Neurosci 31:3894-3903.

Raemaekers M, Bergsma DP, van Wezel RJ, van der Wildt GJ, van den Berg AV (2011) Effects of vision restoration training on early visual cortex in patients with cerebral blindness investigated with functional magnetic resonance imaging. J Neurophysiol 105:872-882.

Rokem A, Silver MA (2010) Cholinergic enhancement augments magnitude and specificity of visual perceptual learning in healthy humans. Curr Biol 20:1723-1728.

Romanska A, Rezlescu C, Susilo T, Duchaine B, Banissy MJ (2015) Highfrequency transcranial random noise stimulation enhances perception of facial identity. Cereb Cortex 25:4334-4340.

Sabesan R, Barbot A, Yoon G (2017) Enhanced neural function in highly aberrated eyes following perceptual learning with adaptive optics. Vision Res 132:78-84.

Sagi D (2011) Perceptual learning in vision research. Vision Res 51: $1552-1566$

Schwartz S, Maquet P, Frith C (2002) Neural correlates of perceptual learning: a functional MRI study of visual texture discrimination. Proc Natl Acad Sci U S A 99:17137-17142.

Schwarzkopf DS, Silvanto J, Rees G (2011) Stochastic resonance effects reveal the neural mechanisms of transcranial magnetic stimulation. J Neurosci 31:3143-3147.

Sehm B, Schnitzler T, Obleser J, Groba A, Ragert P, Villringer A, Obrig H (2013) Facilitation of inferior frontal cortex by transcranial direct current stimulation induces perceptual learning of severely degraded speech. J Neurosci 33:15868-15878.

Seitz A, Watanabe T (2005) A unified model for perceptual learning. Trends Cogn Sci 9:329-334.

Seitz AR, Dinse HR (2007) A common framework for perceptual learning. Curr Opin Neurobiol 17:148-153.

Silvanto J, Muggleton N, Walsh V (2008) State-dependency in brain stimulation studies of perception and cognition. Trends Cogn Sci 12:447-454.

Snowball A, Tachtsidis I, Popescu T, Thompson J, Delazer M, Zamarian L, Zhu T, Cohen Kadosh R (2013) Long-term enhancement of brain function and cognition using cognitive training and brain stimulation. Curr Biol 23:987-992.

Tan DT, Fong A (2008) Efficacy of neural vision therapy to enhance contrast sensitivity function and visual acuity in low myopia. J Cataract Refract Surg 34:570-577.

Terney D, Chaieb L, Moliadze V, Antal A, Paulus W (2008) Increasing human brain excitability by transcranial high-frequency random noise stimulation. J Neurosci 28:14147-14155.

Tyler SC, Contò F, Battelli L (2018) Rapid Improvement on a Temporal Attention Task within a Single Session of High-frequency Transcranial Random Noise Stimulation. J Cogn Neurosci 30:656-666.

Vaina LM, Soloviev S, Calabro FJ, Buonanno F, Passingham R, Cowey A (2014) Reorganization of retinotopic maps after occipital lobe infarction. J Cogn Neurosci 26:1266-1282.

van der Groen O, Wenderoth N (2016) Transcranial random noise stimulation of visual cortex: stochastic resonance enhances central mechanisms of perception. J Neurosci 36:5289-5298.

van der Groen O, Tang MF, Wenderoth N, Mattingley JB (2018) Stochastic resonance enhances the rate of evidence accumulation during combined brain stimulation and perceptual decision-making. PLoS Comput Biol 14:e1006301.

Wang R, Wang J, Zhang JY, Xie XY, Yang YX, Luo SH, Yu C, Li W (2016) Perceptual learning at a conceptual level. J Neurosci 36:2238-2246.

Watanabe T, Sasaki Y (2015) Perceptual learning: toward a comprehensive theory. Annu Rev Psychol 66:197-221.

Watson AB, Pelli DG (1983) QUEST: a Bayesian adaptive psychometric method. Percept Psychophys 33:113-120.

Yang T, Maunsell JH (2004) The effect of perceptual learning on neuronal responses in monkey visual area V4. J Neurosci 24:1617-1626.

Yan Y, Rasch MJ, Chen M, Xiang X, Huang M, Wu S and Li W (2014) Perceptual training continuously refines neuronal population codes in primary visual cortex. Nat Neurosci 17:1380-1387.

Zoefel B, Davis MH (2017) Transcranial electric stimulation for the investigation of speech perception and comprehension. Lang Cogn Neurosci 32:910-923. 